García-Cárdenas, P.A. y Moreno-Baptista, C. (2019). Aproximación a las formas de representación socio-espacial en personas con diversidad funcional visual en Manizales (Colombia). Revista de Antropología y Sociología: VIRAJES, 21(1), 151-179. DOI: 10.17151/rasv.2019.21.1.7

\title{
Aproximación a las formas de representación socio-espacial en personas con diversidad funcional visual en Manizales (Colombia)*
}

\author{
PAULA ANDREA GARCÍA CÁRDENAS** \\ CESAR MORENO BAPTISTA***
}

Recibido: 27 de febrero de 2019

Aprobado: 2 de abril de 2019

Artículo de Investigación

\footnotetext{
* Este artículo se fundamenta en la investigación de Paula García para optar al título de Antropóloga en la Universidad de Caldas.

** Antropóloga. Universidad de Caldas, Manizales, Colombia. E-mail: pagarciac2007@gmail.com. (D) ORCID: 0000-0003-0265-7260. Google Scholar

*** Doctor en Antropología. Universidad de Caldas, Manizales, Colombia.

E-mail: cmorenobaptista@gmail.com. (1) ORCID: 0000-0002-6283-7952. Google Scholar.
} 


\title{
Resumen
}

El objetivo del presente artículo es hacer una descripción interpretativa sobre el fenómeno de la Diversidad Funcional Visual -DFV- en la ciudad de Manizales, a partir de las habilidades desarrolladas por las personas invidentes para movilizarse en los diferentes espacios en los que desarrollan su vida cotidiana. La investigación es de corte etnográfico, el cual permitió tener un acercamiento con la población con DFV de la Asociación Abre tus Ojos. Se ha querido privilegiar la perspectiva émica con personas que han adquirido la Disfuncionalidad Visual y su proceso de readaptación al mundo cotidiano en la ciudad. Los resultados permiten identificar un recorrido de diferentes situaciones y procesos individuales y familiares, a los que se ven enfrentados las personas desde que experimentan la pérdida de la visión de manera progresiva a lo largo de su vida, hasta lograr un nivel de autoreconocimiento y aceptación de la DFV en sus vidas. Finalmente, se concluye que en el fenómeno de la DFV el conjunto de percepciones, interpretaciones e historias de la vida personal constituyen una realidad poco estudiada en nuestro medio y sobre la cual se debe seguir analizando teniendo en cuenta una mirada más inclusiva en las políticas públicas.

Palabra clave: Diversidad Funcional Visual, memoria sensorial, mapas mentales, inclusión social, lógicas cognitivas.

\section{Approach to the forms of socio-spatial representation in people with visual functional diversity in Manizales (Colombia)}

\begin{abstract}
The objective of the article is to make an interpretative description of the phenomenon of the Visual Functional Diversity -VFD- in the city of Manizales, based on the skills developed by visually impaired people to move around in the different spaces in which they develop their daily lives. This is an ethnographic research with VFD members of the Abre tus ojos (Open your eyes) Association. The purpose has been to privilege an emic perspective with people with Visual Dysfunctionality and their process of readjustment to the everyday world in the city. The results allowed identifying routes through different situations and individual and family processes, which people face when they experience progressive loss of vision until they achieve a level of self-recognition and acceptance of the VFD condition. Finally, it is concluded that in the phenomenon of the VFD, perceptions, interpretations and personal life stories constitute a little studied reality in our environment that must continue to be analyzed taking into account a more inclusive view of public policies.
\end{abstract}


Key words: Visual Functional Diversity, sensory memory, mental maps, social inclusion, cognitive logic.

\section{Introducción}

$\mathrm{E}$ n esta investigación se adopta el concepto de Diversidad Funcional, propuesto en el año 2005 por los autores Javier Romañach y Manuel Lobato en el Foro de la vida independiente realizado en España. El término diversidad funcional lo plantean los autores como una alternativa frente a la acepción oficial de "personas con discapacidad"1. En términos generales, permite asumir literalmente "la realidad en la cual una persona funciona de manera diferente o diversa de la mayoría de la sociedad"2 (Romañach y Lobato, 2005, p. 4). Se refieren específicamente a partes del cuerpo (P. Ej. ojos, oídos, piernas, cerebro, etc.) y también a las funciones que realizamos habitualmente los seres humanos como seres vivos (por ejemplo desplazarse, ver, comunicarse, etc.).

Para nuestro caso las personas con Diversidad Funcional Visual (DFV) corresponden a aquellas que por diversas razones carecen del sentido de la vista y que, por lo tanto, son personas que funcionan de manera diferente que las demás.

El trabajo pretende realizar un análisis descriptivo del fenómeno de la Diversidad Funcional Visual (DFV) en la ciudad de Manizales, a fin de argumentar que las personas que han adquirido la disfuncionalidad desarrollan la habilidad para crear mapas mentales fundamentados en lógicas cognitivas, que les permite adaptarse e interactuar en distintos espacios físicos y sociales de la ciudad, sin el sentido de la vista. Dicha adaptación consiste en el reaprendizaje del uso de sus sentidos restantes y potenciación de la memoria a lo largo de su vida. En términos

\footnotetext{
${ }^{1}$ La Diversidad Funcional (DF) no tiene nada que ver con la enfermedad, la deficiencia, la parálisis, el retraso ni con significados asociados a la incapacidad, el impedimento, la inhabilidad o la deficiencia (entre otros) que tienen una carga estigmatizante y/o excluyente para las personas que han perdido algún sentido.

${ }^{2}$ Diversidad: "Con esa palabra queremos reflejar exactamente eso, la diferencia, la desemejanza con lo que es habitual con la mayoría de la estadística de la especie humana. La palabra funcional, perteneciente o relativo a las funciones; y la acepción función, se utiliza como la capacidad de actuar propia de los seres vivos y de sus órganos, y de las maquinas o instrumentos" (Romañach y Lobato, 2005, p. 6).
} 
de la antropología de los sentidos, se infiere de manera analítica una correlación entre la percepción, la interpretación y la historia personal en el fenómeno.

El interés por esta temática surge de la necesidad personal y profesional de develar una realidad invisibilizada como lo es el fenómeno de la DFV. Los sujetos de estudio a los cuales nos referimos poseen una experiencia de vida compartida al adquirir la pérdida del sentido de la vista con sus implicaciones en las relaciones interpersonales y sociales en la cotidianidad.

Los resultados de la investigación presentan una descripción interpretativa de las dinámicas de los contextos sociales urbanos y de las lógicas internas (subjetivas) sobre el fenómeno de la DFV a través del uso de nociones como: antropología de los sentidos, representaciones sociales, el concepto de propiocepción, memoria sensitiva y sociografía multisensorial.

\section{Contextualización de la población sujeto de estudio}

Según el Departamento Nacional de Estadística (DANE), la ciudad de Manizales registra en el año 2016 una población de 397.466 habitantes, de los cuales el $93 \%$ viven en el área urbana y el restante $7 \%$, en el área rural (Diario Eje, 2016, p. 1). De este número de habitantes según el Registro de Localización y Categorización de Personas con Discapacidad (RLCPD) actualizado hasta junio 2015, en Manizales se encuentran registradas 7.065 personas que presentan alguna "Diversidad Funcional" que puede ser motora, cognitiva, sensorial visual, sensorial auditiva. Entre las cuales 397 presentan Diversidad Funcional Visual. Para nuestro trabajo, la población sujeto de estudio fueron los integrantes con DFV de la Asociación Abre tus Ojos, fundada el 8 de noviembre del año 2016, que actualmente cuenta con 89 afiliados, y su sede se encuentra en la Casa de La Cultura del barrio San José (Manizales/Caldas). Esta asociación en los casi dos años que lleva de estar constituida, se ha interesado en ofertarle a sus miembros capacitaciones y talleres para facultarlos en ciertas habilidades como la movilidad, entre otros. Con este grupo elegido para desarrollar la investigación se indagó sobre las estrategias que tienen estas personas para desarrollar un espacio físico vital, a partir de vincular su experiencia de vida a percepciones sensoriales y significados en mapas mentales o representaciones de los espacios cotidianos y urbanos.

El trabajo etnográfico de la investigación se sitúa en una perspectiva émica, en la que la investigadora con DFV hace parte de la comunidad de estudio. Así es que la observadora realiza una auto-etnografía o reflexividad 
al situarse frente al "espejo" y analizar su propia experiencia personal en el análisis de la DFV en Manizales.

Desde el punto de vista epistemológico, el trabajo de campo significó hacer etnografía en la propia comunidad del observador; lo que algunos antropólogos llaman trabajos "en casa", o estudios sobre "nosotros", poniendo el acento en las importantes posibilidades que esta opción puede ofrecer. En este caso, la observadora que pertenece al grupo objeto de estudio tuvo una particular libertad de movimiento y una posición privilegiada de observación de los "otros", pudiéndose relacionar con todas las personas de su grupo a diferentes niveles de confianza para hablar sobre la experiencia de vida con todas y cada uno de ellas en relación con el tema de la DFV. En el marco de las actividades cotidianas y la interacción con los pares con DFV, se aplicaron las entrevistas semiestructuradas en las cuales se abordaron las temáticas que se exponen en los contenidos del texto como: la relación con la familia, la asimilación de la perdida de la visión, las estrategias de aprendizaje para los desplazamientos en los espacios del hogar y la ciudad. La observación en el propio grupo le ofreció, además, mayor posibilidad de acceso a la vida cotidiana en la que viven personas con DFV y la garantía de mayor información al respecto.

\section{Descripción del proceso}

Los resultados de la investigación hacen una descripción interpretativa de los recorridos experimentados por las personas que adquieren la DFV en contextos sociales urbanos y de las lógicas internas (subjetivas) sobre el fenómeno de la DFV. Desde nociones como: antropología de los sentidos (Le Breton, 2007), representaciones sociales (Goffman, 2009), el concepto de propiocepción (Hafelinger y Schuba, 2010), memoria sensitiva (Gratacós, s.f.) y sociografía multisensorial (Colmenares y Romero, 2005), nos aproximamos a las personas con DFV para tratar de conocer las formas propias de hacer las cosas y recrear mundos posibles en la vida cotidiana.

La Diversidad Funcional Visual, como se ha dicho, consiste en el hecho de que una persona carece del sentido de la vista por razones congénitas (de nacimiento) o adquirida en algún momento de la vida bien sea por una enfermedad o algún traumatismo que afecte el funcionamiento normal del órgano visual. Las causas por las que se puede tener DFV son muy variadas.

Para nuestro caso tomaremos en cuenta la DFV adquirida que es la característica de la población con la cual se ha tenido un mayor acercamiento. Estos casos se presentan generalmente por patologías que inducen un proceso degenerativo del órgano visual o la perdida inmediata de la 
visión. También existen los casos en que la patología no está directamente relacionada con el órgano pero lo afecta indirectamente como el caso de uno de los testigos que por efectos de un tumor en la glándula hipófisis, que hizo compresión sobre el nervio óptico, perdió la visión.

Además de los factores patológicos como los tumores, el glaucoma, enfermedades genéticas o el no desarrollo de algunas células, también se presentan enfermedades degenerativas, donde el perder la visión se debe a un proceso donde la capacidad de ver se va disminuyendo lo que produce que en algún momento se presente DFV ya sea por medio de la ceguera o la baja visión.

En el proceso de la enfermedad las personas recurren a diferentes alternativas médicas buscando reducir el menor daño posible, en algunos casos se logran resultados positivos pero en otros el daño es irreversible. También hay casos donde las patologías van deteriorando progresivamente la capacidad de visión a medida que van pasando los años: “Mi mamá me cuenta que cuando yo tenía 4 años notó que yo tenía dificultad para agarrar las cosas más o menos hasta los 8 o 9 años que ya visité nuevamente al oftalmólogo y pues ya había disminuido un poco más la visión.... Ya después de los 11 a los 39 años he notado un deterioro en la visión, y en los últimos 5 o 7 años mucho más" (Felipe Franco, comunicación personal, 1 de noviembre de 2017). Las personas con este tipo de patologías tienen baja visión a lo largo y gran parte de su vida, ya que el efecto se da de manera progresiva.

\section{Perdiendo la visión, implicaciones personales}

El perder el sentido de la vista se puede experimentar de varias maneras; el estar en esta situación significa para el individuo que la vive no poder efectuar actividades que realizaba en su vida cotidiana con normalidad, al menos en una etapa inicial seguida a la pérdida de la visión, ya que al no tener una percepción visual de lo que lo rodea, el individuo se siente limitado para realizar las actividades con seguridad. "No podía hacer el trabajo habitual que hacía en el computador y en el televisor, se me dificultaba bastante" (José Ancízar Martínez, entrevista personal, 19 de septiembre de 2017).

Empecé a perder visión nocturna y detalles de las cosas, ya no distinguía rostros, ni animales de frente, tampoco veía subidas ni bajadas, tampoco veía los andenes, entonces no distinguía niveles en el día, no distinguía el dinero. (Ancízar Martínez, entrevista personal, 19 de septiembre de 2017) 
Yo ya tenía que andar prendida de mis padres, porque yo ya no era capaz de ver las escalas para subir o bajar, yo ya no escribía con claridad. Escribía con garabatos, entonces yo ya ni podía leer lo que escribía y nadie me entendía las letras. (Carolina Loaiza, entrevista personal, 21 de septiembre de 2017)

La pérdida progresiva de la visión afecta la autonomía de las personas, pues se enfrenta a la dificultad de no poder desarrollar sus quehaceres laborales, domésticos, educativos y públicos con independencia. Por tanto, esta dificultad trae consigo en una primera etapa, un cambio en la forma de realizar las actividades cotidianas. En el hogar se empieza a tener que buscar la forma de resolver situaciones domésticas como el reconocimiento de espacios y la realización de tareas personales como preparar los alimentos, el aseo personal, etc. Actividades como salir a la calle, realizar un compromiso en un lugar público, visitar a otra persona, abordar el transporte público y manejar dinero se van haciendo imposible de realizar sola.

Esta condición también tiene implicaciones en otros ámbitos de la persona como en el educativo, por las limitaciones para leer y escribir, lo que por lo general termina en el abandono de los estudios, como señala uno de los entrevistados:

Yo estaba en el colegio, pero yo noté que la baja visión que tenía iba empeorando con el tiempo, hasta el punto de que ya no podía escribir, ya no podía estudiar, porque no veía la hoja para escribir, y pensé en que tenía que retirarme. (Carolina Loaiza, entrevista personal, 21 de septiembre de 2017)

La percepción del tiempo se modifican "Los horarios que manejaba yo para llegar a mi casa, todos los horarios que manejaba toda mi vida cambiaron, significó dejar de trabajar por el tipo de oficio" (Eucario Ramírez, entrevista personal, 6 de septiembre de 2017).

Como resultado, todas estas transformaciones a las que se ve sometido el individuo con DFV, van a originar un impacto a nivel personal que influye en la manera en que se percibe a sí mismo. Los cambios en el modo de vida provocan en el individuo con DFV cierto grado de incertidumbre:

A nivel personal es angustiante, hay una ruptura en cuanto a lo que uno venía haciendo, por ese cambio de hábitos, uno cambia lo que venía haciendo, y eso genera mucha incertidumbre y angustia, ya que uno se pregunta ¿Qué podrá venir en un futuro?. (Ancízar Martínez, entrevista personal, 19 de septiembre de 2017) 
La pérdida de la visión produce una sensación de incertidumbre frente a la realidad por la falta del sentido de la vista. Se generan muchos interrogantes que van desde los más prácticos hasta lo más trascendente, y desde las cosas más íntimas hasta la vida en sociedad. Así es que surgen sentimientos de culpa que se ponen de manifiesto en expresiones tales como:

¿Por qué tenía que pasarme esto a mí?, si yo no he sido malo, dentro de lo normal soy buena gente. Surge en la persona una sensación de inutilidad y dependencia. Yo pensé que perder la visión era depender exclusivamente de alguien que me llevara, que me trajera,... que mi vida estaba condicionada a lo que alguien quisiera hacer por mí. (Eucario Ramírez, entrevista personal, 6 de septiembre de 2017)

Se produce una baja en el autoestima, el estado de ánimo se debilita, se siente vergüenza al pensar que las otras personas se enteren de su ceguera, "esa fue una depresión muy grande, tan grande que duré un año prácticamente sin salir de mi casa, porque me daba pena que vieran que yo no veía" (Gustavo Adolfo Vargas, entrevista personal, 6 de octubre de 2017). Los testimonios de los entrevistados de la Asociación Abre tus Ojos coinciden en señalar que en un primer momento les significó estar fuera de los parámetros establecidos por la sociedad, lo que de alguna manera los llevo a sentirse como alguien "impropio" o poco útil.

Esta sensación de incertidumbre en la vida cotidiana y angustia frente a la noción de futuro, hace que la persona empiece a cuestionarse y enfrentarse al Sí mismo, a tener que repensarse y a tener que "rehistorizar" su vida personal.

\section{La familia}

Así como el individuo que adquiere la DFV a nivel personal experimenta sensaciones de inseguridad, con el grupo familiar sucede algo similar. Cuando uno de los integrantes de la familia pierde el sentido de la visión, sus miembros empiezan a percibirlo como alguien muy vulnerable que no puede valerse por sí mismo y, por lo tanto, todo lo que lo rodea se convierte en una amenaza. Esa sensación hace que se desarrolle un comportamiento sobreprotector vigilante y controlador hacia cada una de las actividades de la persona, lo que a la postre trae un efecto negativo en el estado anímico de la persona, pues consciente o inconscientemente se refuerza la sensación de inseguridad. 
De otro lado, en el colectivo familiar se vive un efecto de baja autoestima o vergüenza: "Según mi percepción, uno se convierte en dos tipos de persona: la primera, se vuelve como una porcelana, que si se mueve se puede quebrar; y la segunda, es una persona que no se debe mostrar" (Eucario Ramírez, entrevista personal, 6 de septiembre de 2017). Se trata de una impresión negativa que se despierta asociada a la idea de ser alguien inadecuado y, en consecuencia, la necesidad de tener que ocultarse. Es una impresión que aparece con la idea que "nos ven, o pueden vernos" que se considera afecta negativamente la imagen de nuestra apariencia, "una persona con discapacidad en la casa, que pena que la gente se dé cuenta, o que pena sacarla a la calle" (Eucario Ramírez, entrevista personal, 6 de septiembre de 2017), señala uno de los testimonios.

Esta actitud de los miembros de la familia también refuerza los desasosiegos iniciales del invidente, pues contribuyen a que se perciba a sí mismo como alguien que no debe estar en contacto con la sociedad. Los pensamiento negativos asociados a la idea de ser defectuoso, no ser como los demás, ser incompetente, no ser querido, ser débil, ser inútil, acrecienta el sentimiento de retraimiento y genera un sufrimiento en el individuo y el colectivo familiar (Bau, 2016). Socialmente surge el temor de tener que "enfrentar donde quiera que vaya la situación de ser recibido como alguien que ya no es más lo que algún momento fue" (Goffman, 2010, p. 95).

Se podría inferir que esta condición puede responder a los prejuicios morales de nuestra sociedad sobre los cuales se fabrican ciertos estándares sociales, biológicos y culturales y se establece una suerte de estructura biográfica única (Goffman, 2010). De tal forma que por diferentes circunstancias un individuo que está por fuera de alguno de estos patrones sienta temor a ser estigmatizado.

\section{Aceptando la Diversidad Funcional Visual}

La sensación inicial de no tener la capacidad de hacer nada cuando se adquiere la DFV, se debe en gran medida a que es una situación nueva que tanto el individuo como su entorno familiar no sabe o no está preparado para afrontar, además de pensar que será una situación permanente e indefinida. Se comprende porque tanto el individuo como la familia se encuentran experimentando un suceso en su vida que ha causado un gran impacto y, a razón de esto, se han producido muchas modificaciones en la vida diaria, laboral, educativa, etc., que en la mayoría de los casos no se está preparado, ni se tienen las condiciones o el conocimiento suficiente para sobrellevar este escenario. 
En los casos donde la DFV se adquiere a temprana edad, la familia busca alternativas con el fin de encontrar una posibilidad que le permita al individuo superar este episodio, como señala uno de los entrevistados refiriéndose a sus padres: "Ellos buscaron inicialmente pues ayudas médicas, me llevaron a muchas clínicas... después de buscar todas esas posibles soluciones, les dijeron que no había solución para que yo viera, o para que yo tuviera algo de visión" (Sergio Bermúdez, entrevista personal, 31 de octubre de 2017).

Como se ha resaltado anteriormente la Diversidad Funcional Visual no es una condición que solo afecte al individuo que la presenta, sino que también afecta emocionalmente a las personas más allegadas. Sin embargo, la forma en como se desarrolle la capacidad de asimilación de la situación, será determinante en el proceso de readaptación del individuo y la familia.

El aceptar la condición de la DFV para un individuo representa, primordialmente, el aprender a vivir con la nueva limitación, lo que implica desarrollar el convencimiento que el no poder ver no debe ser un impedimento para vivir en sociedad; como lo señala uno de los entrevistados: "Yo entendí que se puede vivir sin visión, es que la vida no se trata de poder ver, oír, caminar, la vida se disfruta de muchas maneras" (Eucario Ramírez, entrevista personal, 6 de septiembre de 2017). El ser conscientes que tener DFV no es un impedimento para vivir, le permite al individuo reconocer en esta condición un nuevo modo de aprehender la exterioridad que le rodea: "Entendí que la discapacidad visual es un mundo espectacular, porque es un reaprender, un volver a comenzar" (Eucario Ramírez entrevista personal, 6 de septiembre de 2017).

El aceptar la DFV como un nuevo modo de aprender la realidad y hacer parte de ella, repercute en que el individuo desarrolle la necesidad de experimentar con su condición. "En un momento dado empieza uno a querer aprender y a probar estrategias para uno poderse defender lo más normalmente posible" (Ancízar Martínez, entrevista personal, 19 de septiembre de 2017).

Esta necesidad del invidente por experimentar y descubrir estrategias que le faciliten desenvolverse en la vida cotidiana, responde a que la aceptación de la DFV ya no la ve como algo incapacitante sino que comienza a pensarlo como una posibilidad de redescubrirse como individuo. Este redescubrimiento consiste, entre otras cosas, llevar a cabo actividades que antes de adquirir la DFV no las realizaba, como señala uno de las entrevistadas: "Yo aproveché para hacer natación, también entre a clases de musicografía, aproveche para hacer cosas nuevas que me dieran ánimo" (Carolina Loaiza, entrevista personal, 21 de septiembre de 2017). 
Este interés por hacer actividades nuevas en el proceso de aceptación de la condición, reafirma en la persona la búsqueda de esa nueva imagen dejando atrás la sensación de inhabilidad que se adquiere al principio de la nueva situación. Asimismo, la necesidad de sentirse útil por encima de la DFV impulsa en el individuo el interés por desempeñar ciertas labores que le representen un reto a superar como una forma de fortalecer la convicción de demostrarse a sí mismo y a los demás sus capacidades para llevar una vida normal a pesar de las limitaciones visuales, además de que está en un proceso de aceptación de reconocerse como alguien diferente, pero no en términos negativos o incapacitantes, sino como alguien con una característica diferente que le permite conocer y explorar su realidad de diversas maneras.

Asimismo, el decidirse a elaborar diferentes oficios es un indicador de que ya se ha alcanzado una conciencia donde se comprende que la DFV es algo que hace parte de la realidad del individuo, pero que esa realidad no es una razón para limitarse en el desempeño de diferentes ocupaciones: "Yo comencé con la intriga de querer hacer ya pues mi vida, ya normalizar pues... yo ya había asumido que mi ceguera ya era conmigo, que era algo mío" (Orlando Chávez, entrevista personal, 10 de julio de 2017).

Del mismo modo, esta aceptación por parte del individuo con DFV va encaminada a que las personas convencionales reconozcan que el no tener el sentido de la vista no es razón para minimizar a los individuos que presentan esta condición, sino que simplemente lo empiecen a ver y aceptar como alguien diferente, pero no con la carga de la exclusión, sino como un individuo más de la sociedad que tiene las mismas capacidades que los demás.

El querer ser reconocido ante la sociedad solo como una persona con una característica especial significa que el proceso de aceptación de la DFV no solo depende del individuo que posee la condición, sino también de los otros individuos que hacen parte de la sociedad. El lograr este tipo de aceptación le permitirá al individuo considerarse un miembro más de la sociedad. Y esto a su vez se verá reflejado en la forma en que este asuma su modo de ser.

Por otro lado, el aceptar esta nueva condición consiste en comprender por parte del individuo que a raíz de esto van a surgir cambios en las ocupaciones que hacía antes de adquirir la DFV, sin embargo, esto no va a ser impedimento para retomar su cotidianidad. Por consiguiente, el querer retomar la cotidianidad que se tenía antes por el individuo significa que su DFV es más aceptada; que aprendió a reconocer en sí mismo un ser completamente apto para hacer parte de la sociedad, aceptando los cambios ocasionados por esta condición. Por tal motivo el individuo debe buscar 
mecanismos para capacitarse, para poder retomar en gran parte la vida que llevaba, logrando así también alcanzar la autonomía que se puede perder a causa de carecer del sentido de la vista.

Así pues, el experimentar la DFV para una persona implica pasar por diferentes estados a nivel individual, familiar, y/o colectivo, además de preguntarse cómo será reconocido por la sociedad. No obstante, después de vivir estas etapas, el individuo interioriza su estado actual generando la obligación de indagar acerca de los nuevos elementos que quiere incorporar a su vida con la finalidad de capacitarse y así ser un actor más en la sociedad.

\section{Aprendiendo a vivir con la Diversidad Funcional Visual}

Después de que el individuo se apropia de su nueva condición, inicia un proceso en el cual la prioridad es buscar capacitación y nuevos conocimientos para adaptarse a esta nueva etapa de su vida. Así es que, el aprendizaje del sistema de lecto-escritura Braille se vuelve una prioridad para que la persona pueda acceder a diferentes contextos sociales como el ámbito educativo: "Apenas aprendí Braille y ábaco empecé a validar el colegio, simplemente era cambiar mi modo de vida y adaptarla a otras cosas" (Gustavo Adolfo Vargas, entrevista personal, 6 de octubre de 2017). También existe la posibilidad de acceder a nuevas tecnologías que abren posibilidades de ocupación para personas con DFV, por ejemplo en Manizales la Asociación Abre tus Ojos, ha logrado ofrecer cursos de capacitación para personas con DFV, como son los cursos de JAWS (software lector de pantalla para las personas con DFV) ${ }^{3}$, para el manejo de computadores.

En este orden de ideas, se entiende cómo en el proceso de readaptación a la realidad el individuo despierta la sensación de sentirse útil y se abre al uso de nuevos medios que le permitan proponerse y alcanzar nuevas metas personales. Se despierta el ansia por el aprendizaje para realizar diferentes tareas en la vida diaria. Como lo expresa una de las entrevistadas: "A mí me metieron en la rehabilitación a actividades de la vida diaria...o sea, en las labores del hogar como por ejemplo el barrer, trapear, cómo preparar alimentos" (Beatriz, entrevista personal, 7 de noviembre de 2017). Para otras personas la formación académica se constituye en la meta principal.

Como lo plantea Erving Goffman en su texto: La presentación de la persona en la vida cotidiana, de hecho todo individuo está preparado para

\footnotetext{
${ }^{3}$ Este programa les brinda la posibilidad de usar un computador, pues el software permite a través de una aplicación recibir las indicaciones de una voz sintetizada que va describiendo paso a paso las operaciones que la persona va realizando en el computador.
} 
desarrollar habilidades para poder llevar a cabo diferentes oficios y cuidar de su presentación ante los demás.

Así, cuando el individuo va logrando confianza en sí mismo y reconoce su nuevo modo de vivir y su capacidad de adaptarse, también ve la necesidad de recobrar la autonomía para desplazarse por sí solo en la calle y dejar de depender de las otras personas para su movilidad. El encuentro y la interacción con otras personas que poseen su misma condición son muy importantes, ya que a través de este contacto donde se comparten experiencias, tratan por lo común de adquirir información acerca del "otro" y de poner en juego lo que ya poseen. En este sentido, el incentivarse a alcanzar la autonomía que se pierde en un primer momento, puede estar influenciado por la motivación y las metas personales, lo que implica interactuar con diferentes espacios físicos y sociales.

Todo empezó por que yo fui a la universidad y expuse mi deseo por estudiar allá, y el decano de la facultad de Psicología me dijo, es que aquí no te vamos a recibir con una persona que te esté llevando, tú tienes que aprender a movilizarte. (Carolina, entrevista personal, 21 de septiembre de 2017)

El comenzar esta nueva etapa en su vida le exige al individuo superar las circunstancias de limitación visual, siendo una de las necesidades básicas aprender a desplazarse en el espacio de la casa y por la ciudad de manera autónoma. De acuerdo a este reto, el paso a seguir es aprender a movilizarse. Lo que puede lograr de manera empírica o recibiendo capacitaciones en el área de orientación y movilidad, donde puede desarrollar las habilidades y las técnicas para desplazarse solo, con clases particulares. En este aspecto, se requiere que las instituciones del Estado desarrollen políticas de inclusión para personas con DFV, pues actualmente son inexistentes, al menos en la ciudad de Manizales.

El apoyo profesional en la capacitación es fundamental ya que con su conocimiento y sus orientaciones el individuo con DFV puede tomar conciencia de las situaciones en el espacio recurriendo al uso de los demás sentidos: el oído, el tacto, el olfato y el gusto. "Antes de la primera salida, la profesora me hizo un examen en la casa, para ver qué tan buen oído tenía y que tan buenos reflejos" (Orlando Chávez, entrevista personal, 10 de julio de 2017). "En las clases de movilidad y orientación inicialmente se trabaja el equilibrio... también se aprende a caminar en línea recta, con una guía en el piso para que uno se haga la idea de caminar en línea recta" (Sergio Bermúdez, entrevista personal, 20 de agosto de 2017). 


\section{La técnica del bastón guía}

El aprender a caminar en línea recta es de suma importancia, ya que al momento de salir a la calle es importante mantener el desplazamiento por las zonas peatonales como los andenes, y los cruces de los semáforos por la cebra. El paso siguiente, en el aprender a desplazarse independientemente, es desarrollar el uso del "bastón guía". Este será la herramienta que le va a permitir a la persona moverse sola en calle. Aquí cabe anotar que el bastón tiene ciertas características, pues es un bastón guía y no uno de apoyo. El bastón debe tener una medida especial, la cual se toma desde la cabeza del esternón hasta el piso, es decir, que cada persona tiene una medida específica para ser usado adecuadamente. La persona debe llevar el bastón ubicándolo, a partir del punto medio del cuerpo hacia adelante, de tal forma que al palpar el piso la persona dibuje un arco que explore los obstáculos abarcando el espacio correspondiente a la distancia entre hombro y hombro de su cuerpo, lo que le permitirá explorar las características del camino y los posibles obstáculos que pueda encontrar al momento de desplazarse por diferentes lugares. En los desplazamientos es importante la sensibilidad que se desarrolla con el tacto de los pies al contacto con el piso, lo que le permite a las personas identificar las características del piso (por ejemplo, si es un piso liso, escabroso o desnivelado).

La forma como se debe caminar con el bastón es intercambiando los pies para el lado contrario que se desplace el bastón, es decir, cuando el bastón este al lado izquierdo, se da el paso con el pie derecho, y viceversa. Se debe hacer así, para que el bastón explore con anterioridad el terreno por donde se camina y para identificar los obstáculos y poder dar el paso con seguridad. Solo después de aprender la técnica, el individuo puede ensayar las primeras exploraciones para desplazarse por determinados espacios y familiarizarse con el uso del instrumento. "Primero se trabajó en un terreno plano como una cancha, y luego sí se hacían muchos recorridos en la calle para empezar a hacer una exploración, aprender a percibir con el bastón, sentir el terreno, a sentir los obstáculos"4 (Sergio, entrevista personal, 31 de octubre de 2017). Sin embargo, en un primer momento, el enfrentarse al uso del bastón le genera al individuo sensaciones de vergüenza ante los demás, lo que hace que en un comienzo se muestre renuente al uso de este, "A mí me daba pena usar el bastón, que vergüenza. Entonces, por ejemplo, yo llegaba a la esquina, me encontraba con mi mamá y lo primero que hacía era doblar el bastón y guardarlo" (Gustavo Vargas, entrevista personal, 16 de octubre de 2017).

\footnotetext{
${ }^{4}$ Entrevista 1970. Sergio Bermúdez.
} 
Los primeros acercamientos al bastón también producen en la persona con DFV, una sensación de inseguridad, pues se duda que el instrumento sea suficiente ayuda para movilizarse, ya que la persona se va a tener que enfrentar con muchos y variados obstáculos, por ejemplo, huecos, objetos en la vía, personas, etc. Pensar de esta manera obedece a que el individuo ha tenido la experiencia de uso de los cinco sentidos para conocer su medio exterior, y sabe que las condiciones a las que se tiene que enfrentar no son las mismas, por lo que se siente limitado para realizar sus desplazamientos.

De otro lado, el empleo del bastón enfrenta al individuo al reto de aceptar su condición socialmente, presentarse ante los demás, recuperar su autonomía y empezar a construir sus proyectos personales. Con el paso del tiempo, cobra más importancia, el interés de superación el tener la libertad de desplazarse solo, y el bastón cobra un significado de independencia. “Es un proceso el aceptar el bastón claro, por esa pena que te digo, pero cuando uno lo usa ya en todos los espacios, uno aprende a querer el bastón, el amigo inseparable" (Eucario Ramírez, entrevista personal, 10 de agosto de 2017).

\section{Estrategias para crear mapas mentales}

Para continuar con el análisis de la DFV es importante que incorporemos algunos elementos teóricos que nos permitirán aproximarnos a la comprensión de lo que podemos llamar lógicas cognitivas en la construcción de mapas mentales. Desde la perspectiva de la antropología de los sentidos, se plantea que "los sentidos no solo forman parte de procesos biológicos en los seres humanos sino que son construcciones de significados compartidos colectivamente con base en una condición social, cultural, ambiental y con matices en las historias individuales" (Le Breton; 2007, p. 7). Lo que nos permite, de modo particular, pensar la manera de experimentar la corporalidad, las percepciones y las formas de significar o representación de los contextos físicos en las personas con DFV.

De acuerdo con Moscovici (1979) las representaciones son:

Sistema de valores, nociones y prácticas que proporcionan a los individuos los medios para orientarse en el contexto social y material, para dominarlo... un corpus organizado de conocimientos y una de las actividades psíquicas gracias a las cuales los hombres se integran en un grupo o en una relación cotidiana de intercambios, liberan los poderes de la imaginación. (p. 82) 
En este mismo sentido, las representaciones sociales son el resultado de los procesos mentales individuales y colectivos en los que se incluyen las estructuras sociales y culturales, y dichas estructuras producirán un proceso cognoscitivo en el cerebro, donde le otorga ciertas características de significado a esas representaciones. Es decir, que las representaciones socio-espaciales se tomarán como esos referentes a los cuales se les atribuyen ciertos significados comunes e individuales, con los cuales es posible identificar y conocer la ciudad y sus espacios urbanos, orientando y posibilitando ciertas prácticas en esta. Entonces, se puede entender que las representaciones socio-espaciales son el referente mental de algo en particular, en este caso, los diferentes espacios urbanos, además, están compuestas por las interacciones y relaciones que se desarrollan, tanto en contacto con los espacios como con los individuos, con los que se interrelaciona una persona en los distintos lugares de la ciudad.

Las representaciones socio-espaciales estarán estrechamente relacionadas con la experiencia multisensorial de un individuo con DFV, ya que a través de esta experiencia se crearán cada una de las representaciones que tiene. Por lo tanto, en las interacciones realizadas en los espacios urbanos y con los sujetos con los que comparte dichos espacios, sus sentidos serán los medios para recolectar una variedad de información de su entorno y así poder familiarizarse con este. En términos de Le Breton (2007): "Las actividades perceptivas decodifican el mundo circundante y lo transforma en un tejido familiar y coherente" (p. 8).

En este orden de ideas, la propiocepción o percepción del propio cuerpo está unida a patrones de percepción existentes, y está basada en procesos sensomotrices. El cuerpo reacciona a los estímulos externos e internos con movimiento, o adoptando determinadas posiciones. "Todos los movimientos del ser humano tienen lugar en un juego de alternancia, entre los estímulos sensitivos y su aplicación motora" (Hafelinger y Schuba, 2010, p. 1).

Por tanto, una persona con DFV y asumiendo el cuerpo como otro órgano sensitivo, por medio de la propiocepción buscará adquirir una serie de información de su entorno gracias a los diversos estímulos que recibe en las diferentes interacciones en los espacios urbanos, según afirma Merleau-Ponti (1993): “La pura sensación, definida por la acción de los estímulos sobre nuestro cuerpo, es el 'efecto último' del conocimiento, (...), gracias a una ilusión, por otro lado natural, que la colocamos al principio y la creemos anterior al conocimiento" (p. 59). Es decir, la percepción propioceptiva les permite a los individuos procesar el contexto a partir de ciertos códigos e interpretaciones de lo que se haya en el ambiente circundante, ya que por medio de lo que perciba configurará 
unos significados con el fin de adquirir una conciencia de su exterioridad. Por tanto, la propiocepción es un canal de información que da ciertas capacidades al individuo que son requeridas para la supervivencia del ser humano. Así, el sentido propioceptivo que se estimula en las personas con DFV es una herramienta vital para configurar una serie de representaciones del contexto que le dan la oportunidad de converger en el espacio social, poder interactuar con los demás individuos y desempeñar una variedad de actividades.

Diseñar estrategias a partir de las percepciones sensitivas que recopile un sonido, un aroma o la textura de la superficie por donde camine, serán primordiales para interactuar con el mundo que lo rodea. Así, los sentidos (auditivo, táctil y olfativo) constantemente estarán activados, ya que estos le permitirán a una persona con DFV concebir de manera práctica su exterioridad. Así lo manifiesta uno de los entrevistados:

Hablo de mi experiencia. Cuando yo perdí la vista se me empezaron a potencializar los otros sentidos. Tanto que cualquier ruido le lastima a uno el oído, el olfato totalmente desarrollado y el tacto ni se diga, hasta con los pies, porque uno constantemente está palpando la superficie; por ejemplo cuando voy por unos adoquines o está rugoso o pedregoso; o el olfato, me huele a tinto quizá esté llegando a la cafetería de tal parte; o me huele a muebles nuevos, entonces estoy llegando a la mueblería que está llegando al Parque Bolívar; o me huele a peluquería por los secadores, entonces ahí está la peluquería de tal parte. Entonces uno aprende a desarrollar todos esos sentidos para la ubicación. (Eucario Ramírez, entrevista personal, 6 de septiembre de 2017)

Es decir, que cada uno de los estímulos sensoriales que perciben las personas que carecen del sentido de la vista no solo será el resultado de un acto físico o mecánico sino que será un acto más consiente. Con base en sus experiencias personales forjarán mecanismos que le brinden la posibilidad de ser un sujeto social activo.

De acuerdo con lo planteado por Harvey, el espacio se puede experimentar de dos maneras: el espacio perceptual y el espacio simbólico Harvey (como se citó en García, 2015):

El primero comprende la experiencia sensitiva, táctil, acústica, sinestécica; este tipo espacial concibe el esquema o mapa mental como necesario además de integrar por la práctica a la memoria, relacionada con el modo de pensamiento adquirido y el espacio simbólico se 
determina a partir de la experiencia espacial abstracta, permite la interpretación y la representación simbólica. (p. 4)

Bajo estos parámetros, el espacio para los individuos con DFV no solo sería concebido como los elementos físicos que los rodean, sino que sería también todas las percepciones que se obtengan en las interacciones, porque, finalmente, estas son las que le posibilitan conocer y comprender el espacio que lo circunda. Además, a partir de ese espacio perceptual tendrá lugar el espacio simbólico, ya que por medio de lo que él perciba de los diferentes lugares por donde se desplace y también de las interacciones que realice con otros individuos donde le describan un lugar en específico, podrá representarse ese espacio bien sea a través de un mapa mental o una imagen mental, la cual será guardada en su memoria para que por intermedio de esta pueda relacionarse y familiarizarse con un espacio determinado.

\section{Memoria sensorial y espacio}

Siguiendo lo anterior, las lógicas cognitivas implican una experiencia corporal a través de la cual se logra conocimiento, conocimiento que como veremos se logra a través de los canales de la percepción de los sentidos que son memorizados, codificados y/o significados en la interacción permanente con el entorno físico y social.

Se puede definir la memoria sensorial como la permanencia de un estímulo en el tiempo, más allá de su presencia física, es decir, es la memoria que permite que el efecto de un estímulo continúe, aunque este haya desaparecido. La memoria sensorial es una capacidad eumnésica o buena memoria que pone de manifiesto la estrecha relación que guardan los sistemas perceptivos y los procesos cognitivos. De hecho, no se puede explicar el funcionamiento del uno sin el otro, la percepción sin cognición conduciría a un modo pasivo de conectar con el mundo, ya que la mente no realizaría ninguna tarea con la información recolectada por los sentidos.

De este modo, la memoria sensorial procesa y almacena la información significativa. Asimismo, "interviene en procesos básicos de la percepción, tales como la detención, discriminación, el reconocimiento y la identificación" (Gratacós, s.f., p. 1).

En la memoria sensitiva se recopilarán todas las situaciones, sensaciones o datos en los que se vea involucrada la existencia del sujeto, ya que a partir de estos elementos los individuos guiarán su cotidianidad, y esto se debe a que en la memoria sensitiva se retendrá una gran cantidad de información. No obstante, es de mayor relevancia aquella que nos da los 
elementos que nos facilitan actuar en la sociedad. En el caso de las personas con DFV estarán registrando información por intermedio de sus sentidos y su cuerpo constantemente. Por otro lado, el individuo hará una selección de los datos que sean más importantes para otorgarle un significado en su esquema mental, esto se debe a que "cada vez que repetimos los estímulos activamos el mismo circuito neural" (Mouráu y Faría, 2015, p. 3).

Cuando se tiene contacto con un estímulo la memoria sensitiva evoca automáticamente un recuerdo almacenado para ser usado en orientar un comportamiento en el plano social. Lo que es de suma importancia en el caso de las personas que carecen del sentido de la vista, porque al no tener esa percepción visual del entorno, deberán hacer uso de las sensaciones recopiladas para interactuar socialmente.

Se podría pensar que la capacidad que tiene la memoria sensorial para guardar un estímulo es corta, debido a que este solo durará lo que se demore en ser percibido; sin embargo, en el caso de las personas que carecen del sentido de la vista no es así, ya que la información que albergue en esta memoria será el capital para construir sus referencias del medio físico que lo rodea.

Ahora bien, si tomamos el espacio como algo más allá del medio físico, en términos de Daniel Harvey (como se citó en García, 2015):

(...) el espacio comprende la experiencia sensible, sinestésica (o percepción de una misma sensación a través de distintos sentidos), misma que se obtiene mediante el uso de dibujos, croquis y/o mapas mentales que registran la práctica del espacio junto con la experiencia del sujeto, puesto que relaciona lo sensible por medio de la práctica a través de la memoria (pensamiento/cosmovisión), cultural, conjunción que permite señalar que la experiencia espacial genera el espacio simbólico. (p. 533)

Es decir, el espacio aquí no solo comprende los elementos físicos que envuelven al individuo sino que igualmente estará compuesto por la experiencia sensorial que provoca en los sujetos las interacciones que efectúen en los diferentes espacios, además, esto derivará en la elaboración de ciertas representaciones proyectadas, por ejemplo, en mapas mentales.

El espacio tomado como un elemento social está compuesto, principalmente, por las prácticas y relaciones sociales que desarrollen los individuos en su cotidianidad. El espacio en gran medida será el encargado de modelar la realidad de los sujetos que interactúan en él, por las diversas representaciones que se originan y la significación que le otorguen a los 
espacios, también por la red de relaciones e interacciones que serán objetivadas por cada individuo en sus prácticas sociales.

El espacio para las personas con DFV será el escenario donde en gran medida adquirirán el conocimiento de un modo sensible para comprender y dotar de sentido el medio que los circunda, tanto social como físicamente, y por intermedio de las representaciones socio-espaciales, que tendrán mayor relevancia, les ayudará a apropiarse de cierto modo del espacio que habitan.

Finalmente para cerrar el dispositivo interpretativo de los mapas mentales desde la DFV, es importante referenciar lo propuesto por Colmenares y Romero (2005), estos dos antropólogos proponen una nueva técnica de investigación social basada en el materialismo dialéctico que plantea que la realidad existe independientemente de si la conocemos o no, y que cada cosa existente es producto de las causas que la originaron, y no de la interpretación que hagamos sobre ellas. Además, esta técnica emplea los sentidos de la percepción ampliando las posibilidades de acercamiento social y proponeusar el concepto de sociedad para efectuar su análisis. Lo planteado por Colmenares y Romero (2005) sirve para buscar un acercamiento a la población con la cual se interactúa en este trabajo a través de sus distintas percepciones, también para reconocer que, aunque las personas con DFV no tienen una referencia visual de la ciudad en la que conviven, no quiere decir que la representación que ellos se hagan de los diferentes espacios urbanos sea errónea o que no sea válida, ya que como lo plantea esta técnica, la realidad existe independiente de si se conoce o no, y las representaciones de personas con DFV tendrán el mismo valor que las que tiene una persona que ve, porque al igual que estos, estarán compuestas por valores similares, solo que no tendrán el referente visual, lo que significa que los individuos que carecen del sentido de la vista tendrán su propia visión de la ciudad.

\section{Habitando la casa}

Uno de los primeros mapas mentales que una persona con DFV ve la necesidad de elaborar es el del espacio de su casa, ya que este es su entorno vital. Por tal motivo, la creación del esquema mental de la forma en cómo está distribuido el lugar donde reside se hará de manera empírica, bien sea por sí mismo o en compañía de alguna persona de la familia. Este aprendizaje le permitirá reconocer los diferentes lugares y objetos de la casa. Cabe diferenciar entre las personas que estando en el hogar de residencia han perdido la visión, de aquellos que por diferentes motivos desconocen totalmente las características del lugar. 
El caso de Paula García (egresada de antropología de la Universidad de Caldas), es el de alguien que ya tenía cinco años de habitar su vivienda cuando perdió la visión. Ella ya tenía una imagen elaborada de su casa y un conocimiento previo en cuanto a la distribución de los espacios dentro de ella; es decir, que tenía interiorizado una lógica socio-espacial del hogar. No obstante, a pesar de estar en un lugar conocido, al perder la visión debe empezar a realizar sus desplazamientos de manera cautelosa, o en su defecto, en compañía de alguien para evitar tropiezos.

En el proceso de readaptación, inicialmente hace un mapa mental de su casa. Hace un inventario de los lugares que está compuesta y las conexiones entre ellos: cocina, baño, habitaciones, corredores, escaleras, etc. De manera similar lo es para una persona con DFV que llega a vivir en un espacio nuevo. La diferencia es que el reconocimiento, en un primer momento, lo debe realizar en compañía de otra persona.

Una vez se haya hecho un esquema mental de los lugares de la casa, es necesario configurar un orden específico de cada elemento de la vivienda con el fin de memorizar la disposición de cada uno de estos en relación con los espacios. Con este trabajo se busca desarrollar cierto grado de autonomía y agilidad para realizar diferentes tareas de su cotidianidad llevadas a cabo en dichos lugares.

\section{Caminando por el barrio}

Las personas con DFV no solo crean mapas mentales de su casa, también lo hacen de otros lugares que visitarán o de los espacios por donde transitarán o realizarán actividades en su vida cotidiana. En estos espacios la información será proporcionada por otras personas, sobre las características de los lugares, sitios, áreas, etc., además de la información que se logre captar por los otros sentidos (oído, olfato y tacto) durante los recorridos y exploraciones. Todos estos datos van siendo retenidos en la memoria para después utilizarlos como una estrategia para planificar los desplazamientos y la movilidad autónomamente.

Al igual que la casa, el espacio público inmediato que debe ser asimilado es el del barrio donde se habita. Para el caso de las personas que perdieron la vista aprender a movilizarse con la ayuda del bastón guía, el barrio donde viven es uno de los primeros espacios que se explora con la ayuda de este instrumento, para generar confianza y familiarizarse con en el espacio público de manera libre.

Al igual que en el hogar, la persona que ha habitado el barrio con anterioridad a la perdida de la visión, tiene una ventaja sobre el que no conoce el espacio previamente; pues como se ha señalado, el conocimiento 
previo facilita la creación de los esquemas mentales. Sin embargo, en el caso de Paula fue preciso tomar clases de orientación y movilidad con una profesora particular, con el fin de aprender a desplazarse con el uso del bastón guía y poner en práctica ciertas técnicas de movilidad como caminar los andenes, cruzar la calle en la esquina y la identificación de los puntos cardinales.

Una vez teniendo la imagen mental del barrio con la distribución por calles y manzanas, el siguiente paso es la planificación de recorridos entre dos puntos, para lo cual es importante ejercitar la memoria que permita recordar los puntos del barrio en relación con la ubicación de la casa. Paula nos proporciona un ejemplo del recorrido que realiza regularmente para tomar clases del manejo de JAWS (software lector de pantalla para las personas con DFV):

Debía salir de mi casa y dar media vuelta a mano izquierda, y empezar a subir por la calle de mi casa hacia el norte. Claro está, haciendo uso del bastón guía moviéndolo en forma de arco y palpando el borde del andén. Cuando llego a la esquina de la cuadra, volteo a mano izquierda y camino 10 pasos hacia el occidente para cruzar la calle, luego de cruzar, me devuelvo 10 pasos hacia el oriente, llego a la esquina de la cuadra y empiezo a caminar hacia el norte subiendo la calle. Cuando llego a la esquina de esa cuadra, giro a mano izquierda y camino aproximadamente 25 pasos hacia el occidente, para poder cruzar la calle, cruzo la calle y camino hacia el occidente para encontrar la esquina y volteo a mano derecha, y sigo caminando hacia el norte. $\mathrm{Al}$ momento de transitar por el andén de esta calle puedo notar que hay varios establecimientos de comidas, gracias a los olores que emiten las diferentes preparaciones, como por ejemplo, el de la pizzería. Este punto de referencia me sirve para saber que aproximadamente estoy a dos cuadras de la casa de mi compañero. Sigo caminando sobre ese mismo andén, hasta cuando percibo un desnivel por una rampa que hicieron para el ingreso a un garaje, en ese punto sé que puedo cruzar. Luego de cruzar la calle, sigo dirigiéndome hacia el norte, pero hasta llegar a la esquina, allí volteo a mano izquierda y empiezo a descender por esa calle hacia el occidente aproximadamente 30 pasos y llego a la casa donde me dirijo a tomar las clases. (diario de campo, Paula García 30 de agosto 2017)

La descripción que hace Paula de uno de sus recorridos semanales, permite imaginar la cantidad de información y el nivel de detalle que debe registrar en la memoria, sobre los puntos de referencia percibidos del entorno; además de la retentiva en la orientación de los puntos cardinales y 
el uso del olfato y el tacto (a través del bastón guía) en la configuración de los mapas mentales.

\section{De camino a la Universidad}

En ciertos recorridos cotidianos se presentan lugares que no ofrecen mucha información auditiva $u$ olfativa que sirva de referencia para el desplazamiento de la persona con DFV. Es donde se requiere haber desarrollado una sensibilidad especial con el bastón guía pues funciona como una extensión del tacto que permite captar las características del piso, por lo que se vuelve un recurso indispensable en los lugares donde no se cuenta con otra referencia que pueda ser percibida por los otros sentidos. Nuevamente recurrimos a la experiencia de Paula que nos sirve de ejemplo:

Un recorrido que no me ofrecía muchos estímulos auditivos y olfativos al momento de caminar fue el que debía de hacer, en el primer tramo, desde mi casa hasta la Facultad de Ciencias Jurídicas y Sociales de la Universidad de Caldas. Sin embargo, es pertinente mencionar que al igual que mi casa y el barrio donde vivo, este recorrido ya era conocido para mí. Esto porque al momento de perder la vista ya me encontraba estudiando la carrera de Antropología. Por tal motivo, este recorrido lo hacía constantemente cuando asistía a las clases en la Universidad. Pero, a pesar de que este trayecto ya era conocido para mí, fue necesario que en las clases de movilidad que tomé para aprender a movilizarme haciendo uso del bastón, re-aprendiera de nuevo ese recorrido. (diario de campo Paula García 15 de septiembre de 2017)

Comenta Paula que el piso en este recorrido presenta muchas irregularidades, bien sea por las modificaciones que se han hecho por los residentes del barrio, los desniveles entre casa y casa, las tapas de alcantarilla, las zanjas, los huecos, etc., y todas estas particularidades del terreno deben ser identificados y memorizados para sortear las dificultades y lograr un desplazamiento seguro.

El mapa mental del recorrido que ha logrado elaborar Paula para desplazarse de su casa a la universidad (sede Palogrande) está compuesto principalmente por las sensaciones táctiles, que obtiene por medio del bastón guía y la información del contacto de los pies con el piso; es decir, que la sensibilidad táctil se potencializa por intermedio del bastón y los pies para configurar el mapa mental. Cada vez que realiza el trayecto es un aprendizaje que le permite distinguir e identificar nuevas sensaciones a las que le va incorporando significado para enriquecer la información 
sobre la cartografía del recorrido. En términos de Le Breton (2007), "La experiencia táctil nos da información de manera sucesiva, discontinua que avanza austeramente descubriendo poco a poco cada elemento del entorno" (p. 171).

La experiencia táctil que logra una persona con DFV por medio del bastón guía y la ayuda de los sentidos restantes (tacto, olfato, oído y gusto) permite descubrir y reconocer la disposición y particularidades del medio físico que lo rodea de una manera muy elaborada y compleja, que para una persona convencional resultaría imposible.

Después de hacer el mapa mental del recorrido que debía hacer Paula para trasladarse a la Facultad de Ciencias Jurídicas y Sociales, de igual forma que en los otros escenarios, tuvo que hacer un reconocimiento de este edificio, con la finalidad de saber al detalle donde se encuentran ubicados ciertos lugares; por ejemplo, las aulas donde debía asistir a las clases, la biblioteca, la fotocopiadora que está dentro de la sede, al igual que otros sitios de interés como la cafetería y los baños.

Aunque Paula ya estaba familiarizada con dicho edificio, para lograr adaptarse al espacio en las nuevas condiciones fue necesario reaprenderlo desde la DFV. Dicho reaprendizaje lo realizó acompañada de la profesora de orientación y movilidad, quien le fue dando las indicaciones de cómo debía caminar por los pasillos. Asimismo, le iba orientando qué elementos estaban presentes en este lugar y podían ser útiles como puntos de referencia para su ubicación espacial y desplazamiento.

\section{Por las calles de la ciudad}

Como ya se ha señalado, cuando se empiezan a hacer recorridos en las calles, los individuos con DFV constantemente tendrán que albergar una gran variedad de información sensitiva en su memoria, ya que continuamente al entrar en contacto con los diferentes lugares de la ciudad por donde transite, encontrará una serie de datos clave para configurar los mapas mentales que se requiere en sus recorridos.

Conjuntamente deberá conocer la nomenclatura de la ciudad, es decir, cómo están ordenadas las calles y carreras; por ejemplo, en qué dirección aumenta y disminuye (para el caso de la ciudad de Manizales, las calles aumentan hacia el oriente y disminuyen hacia el occidente, y las carreras aumentan hacia el sur, y disminuyen hacia el norte). El que una persona que carezca del sentido de la vista tenga conocimiento acerca de este tema, es de mucha importancia, porque le va permitirle a ubicarse cardinalmente y localizar direcciones y lugares de interés (como el caso del centro de la 
ciudad). Esta habilidad es posible desarrollarla con las clases de orientación y movilidad en el proceso de capacitación.

A diferencia de Paula, los demás miembros de la Asociación Abre tus Ojos que fueron entrevistados manifestaron no haber tenido la oportunidad de recibir capacitación de orientación y movilidad, sino que su conocimiento lo obtuvieron de manera empírica. Algunos de ellos lo hicieron en compañía de algún familiar o conocido que le servía de guía para caminar por las calles y otros fueron guiados por una persona con DFV. En ambos casos se logra un desplazamiento autónomo. No obstante, la gestión de la Asociación Abre tus Ojos ha sido fundamental para que sus asociados puedan recibir entrenamiento profesional con el CRAC (Centro de Rehabilitación para Adultos Ciegos), lo que ha contribuido enormemente en lograr complementar, perfeccionar y enriquecer la manera de movilizarse en sus asociados.

Es importante señalar en este punto, que a pesar de las habilidades y destrezas que desarrollan empíricamente las personas con DFV para lograr la movilidad en la ciudad de manera autónoma, son muchos las barreras que debe sortear por la falta de adecuación del espacio público. "Las barreras son todos aquellos condicionamientos en el entorno de la persona que condicionan el funcionamiento y crean discapacidad" (Romañach y Lobato, 2005, p. 3). Algunas de las barreras que resaltan los testimonios de las personas entrevistadas son:

- Hay vías importantes de la ciudad como la carrera 23 que no tiene andenes, o guías que separen el espacio peatonal de la calzada de los vehículos, lo que provoca que fácilmente una persona con DFV termine transitando por el espacio de los carros.

- En ciertos puntos de la carrea 23 se encuentran ubicados bolardos sobre los andenes, los cuales representan un gran obstáculo para la movilidad de las personas con DFV ya que en muchas ocasiones no se percibe con el bastón y se producen fuertes golpes en las piernas y espinillas.

- La misma carrera 23 es una vía muy transitada y el espacio público está ocupado por puestos de vendedores ambulantes, lo que hace más complejo su circulación.

Sumado a lo anterior, existen otras barreras que se presentan en el diseño de la ciudad o en la adecuación del espacio público como alcantarillas sin tapa, huecos en las vías, teléfonos públicos, entre otros, que no permiten una movilidad segura. 
Todo esto significa que no es suficiente el desarrollo de las habilidades por parte de la persona con DFV para adaptarse a la realidad física sin que las condiciones de su entorno no están garantizadas para poder ser funcional.

\section{Viendo más allá de la vista en condiciones de inclusión social}

En este orden de ideas, hemos querido resaltar que la apropiación del espacio por parte de una persona con DFV está estrechamente relacionada con la interpretación subjetiva que haga él de sus prácticas sociales. Por medio de estas prácticas el individuo irá adquiriendo diversas experiencias que le proporcionarán la información que requiere para aprehender y relacionarse con el entorno social y físico que lo rodea.

Como se ha expuesto, a pesar de que la DFV plantea grandes repercusiones en la vida de las personas que la presentan, estos individuos aprenden a vivir con esta nueva condición adaptándose a su nuevo modo de ser y de vivir en la sociedad. Por consiguiente, las personas con DFV aprenden a dejar atrás los imaginarios que están implícitos sobre ellos, que los rotulan como sujetos faltos de capacidades con el uso de términos como inválido o minusválido, los cuales recaen sobre el individuo con una carga peyorativa, limitante y discriminativa.

Es así que la DFV aunque es una condición que impide a ciertas personas ver por medio de sus ojos, también es una condición que viabiliza a los individuos que la viven, para relacionarse con su entorno con una sensibilidad particular, haciendo uso de sus otros sentidos para poder conocer y hacer parte del mundo externo que lo rodea. Por ende, cuando aceptan su condición surge la necesidad de capacitarse y buscar ciertos mecanismos que posibiliten desplegar nuevas habilidades y actitudes que viabilicen el ser sujetos activos en la sociedad. Por tal motivo, como se ha mencionado más arriba, las personas con DFV se capacitan en áreas como la movilidad, en el sistema de lecto-escritura Braille y en el manejo de las tecnologías como la del software lector de pantalla JAWS, entre otros. Esto, con el fin de alcanzar una autonomía e independencia para lograr un desarrollo en diferentes aspectos de la vida.

No obstante, que el individuo con DFV después de atravesar por diferentes procesos a nivel personal, familiar y social aprenda a reconocerse a sí mismo como un individuo totalmente capaz, que logre superarse y adaptarse a las condiciones de la realidad para poder proseguir con su vida y alcanzar un desarrollo en diferentes aspectos de esta, no excluye la responsabilidad del Estado. Es decir, el Estado debe garantizar la posibilidad de accesibilidad para que una persona con DFV tengan las 
mismas oportunidades y la posibilidad real y efectiva de acceder, participar, relacionarse y disfrutar de un bien, servicio o ambiente, junto con los demás ciudadanos.

Es obligación de las administraciones locales que incluyan dentro de las políticas públicas el enfoque diferencial, es decir, medidas efectivas para asegurar que se adelanten acciones ajustadas a las características particulares de las personas o grupos poblacionales, y que garantice el ejercicio efectivo de sus derechos acorde con necesidades de protección propia y específica ${ }^{5}$.

Lamentablemente en la ciudad de Manizales a cinco años de promulgada la Ley 1618 de 2013 por medio de la cual se garantizan los derechos de las personas con discapacidad, los avances en esta política son muy pobres o nulos en la mayoría de los principios y conceptos que definen la misma: comunicativas, físicas, de rehabilitación funcional, rehabilitación integral, en pedagogía actitudinal. Es obligación de las entidades territoriales dar acompañamiento y formación a las familias con DFV, que los programas de compensación implementen programas de inclusión y de información de sus derechos. Igualmente que el municipio junto con las entidades departamentales respalden el trabajo de las redes de apoyo y las organizaciones que buscan el cumplimiento de sus derechos. Asimismo, proveer los servicios de apoyo en la educación para las personas con DFV. Emprender junto con las universidades el apoyo de las investigaciones que permitan comprender el fenómeno de la DFV y trazar estrategias de inclusión social para garantizar la accesibilidad a las personas con mayores dificultades.

\section{A modo de conclusión}

Tomando en cuenta todo lo que se ha expuesto, se puede decir que experimentar el fenómeno de la DFV para una persona implica atravesar por diferentes momentos y etapas de su vida que los cuestiona sobre el sentido de la misma; experiencia que involucra igualmente al grupo familiar.

A lo largo del artículo se ha querido mostrar que las personas que adquieren DFV experimentan un proceso de readaptación a la vida física y social de la ciudad que implica desarrollar lógicas de sentido socio-espaciales

\footnotetext{
${ }^{5}$ Congreso de la República de Colombia, Ley estatutaria 1618 de febrero 27 de 2013 (p. 26): Como manifestación directa de la igualdad material y con el objetivo de fomentar la vida autónoma e independiente de las personas con discapacidad, las entidades del orden nacional, distrital y local garantizarán el acceso de estas personas en igualdad de condiciones al entorno físico, al transporte, (.), el espacio público, los bienes públicos, los lugares abiertos al público y los servicios públicos, tanto en zonas urbanas y rurales.
} 
propias, es decir, formas de correlacionar percepciones, interpretaciones e historias de la vida personal, sumergidos en un mundo de continuidad sensorial siempre presente, donde los sentidos restantes, el tacto, el olfato y el oído se constituyen en el medio principal para interactuar con el contexto.

La descripción que hemos realizado se circunscribió, como lo plantea Le Breton (2007) a las “lógicas de la humanidad (...) que reúnen a los hombres de las sociedades diferentes en su sensibilidad frente al mundo" (p. 7). En nuestro caso, la experiencia en el mundo urbano de las personas con DFV.

Al darle una relevancia particular a los demás sentidos como el oído, el tacto y el olfato, para conocer y relacionarse con el entorno, se rompe en cierta forma con el modo convencionalmente aprendido en las formas de socialización. Para el caso de las personas con DFV explorar otras alternativas que les permita el conocer y el relacionarse con el entorno, los impulsa a prestar mayor atención a los otros canales sensoriales como lo plantea Le Breton (2007): "La educación de los sentidos hace brotar lo múltiple a partir de lo que antes parecía unívoco y simple" (p. 27).

La experiencia de vida en la ciudad de una persona con DFV consistirá principalmente en una construcción de significados y mapas mentales a través de los sentidos del tacto y el olfato (el gusto también), con el fin de lograr aprehender el medio físico que lo rodea. Toda experiencia que tenga estará basada en su modo de sentir y significar el medio físico.

La vivencia propia de las persona con DFV evidencia una circunstancia subjetiva y objetiva acerca de cómo la sociedad percibe a estos individuos que parecen olvidados. Asimismo, como a nivel personal se vive de cierta manera un drama por el cambio en su vida al perder el sentido de vista e, igualmente, la manera como son percibidos. Hasta que aceptan y asumen esta situación y buscan alternativas para capacitarse, superarse y seguir adelante. Con base en la misma experiencia, las personas con DFV empiezan a movilizarse haciendo uso de sus recursos sensoriales para adaptarse al entorno físico que habitan y así alcanzar una autonomía e independencia.

Finalmente, lo expuesto permite constituirse en un llamado de atención a los entes institucionales que administran las políticas públicas a responder con su obligación de atender con políticas inclusivas la población con DFV, haciendo más accesible el espacio urbano a estas personas, pero también con programas de accesibilidad a la educación y a la vida laboral, cultural, etc.

Desde la perspectiva antropológica, el desarrollo de este trabajo busca dar visibilidad a la población con DFV, particularmente de la ciudad de Manizales, de manera positiva revelando su realidad. Contribuir en ese mismo sentido a no subestimar sus capacidades humanas sino salir del 
ostracismo en que se encuentran. Finalmente la investigación se propone como punto de partida para el desarrollo de nuevos trabajos.

\section{Referencias}

Bau, S. (2016). La vergüenza, emoción escondida. Recuperado de https://amadag.com/laverguenza-emocion-escondida/.

Colmenares, J. Á. y Romero, Á. M. (2005). Sociografía Multisensorial. Revista Inversa, (1)1, 23-38.

Crecimiento Lentísimo de la Población en Manizales (2016). Diario Eje. p. 1.

García, I.C. (2015). Apuntes para una Antropología del Espacio. Consideraciones desde la geografía clásica a la geografía cultural. Revista de Antropología Experimental, 28. Recuperado de http://revistaselectronicas.ujaen.es/index.php/rae.

Goffman, E. (2009). La presentación de la persona en la vida cotidiana. Buenos Aires, Argentina: Amorrortu.

Goffman, E. (2010). Estigma, la identidad deteriorada. Buenos Aires, Argentina: Amorrortu.

Gratacós, M. (s.f.). Memoria sensorial: características, funciones y tipos. Recuperado de https:// www.lifeder.com>.

Hafelinger, U. y Schuba, V. (2010). La coordinación y el entrenamiento propioceptivo. Madrid, España: Paidroti.

Le Breton, B. (2007). El sabor del mundo. Una antropología de los sentidos. Buenos Aires, Argentina: Nueva Visión.

Merleau-Ponty, M. (1993). Fenomenología de la Percepción. Barcelona, España: Planeta Agostini. Moscovici, S. (1979). El psicoanálisis, su imagen y su público. Buenos Aires, Argentina: Buemal. Mouráu, C. A. y Faría, N. C. (2015). Memoria. Psicología.: Reflezáo e crítica, 28 (4), 780- 788. DOI: $10.1590 / 1678-7153.201528416$

Romañach, J. y Lovato, M. (2005). Diversidad Funcional, nuevo término para la lucha por la dignidad en la diversidad del ser humano. Recuperado de http://forovidaindependiente. org/wp-content/uploads/diversidad_funcional.pdf 